\title{
Directional Material Probe for Deposition Layer Studies
}

\author{
Suguru MASUZAKI \\ National Institute for Fusion Science, 322-6 Oroshi, Toki 509-5292, Japan \\ (Received 21 May 2013 / Accepted 6 June 2013)
}

\begin{abstract}
A simple new tool for deposition layer studies, a directional material probe, is proposed. The probe, which examines the directionality of deposition layer formation, consists of a flat disk and pin. If deposits have directionality, a shadow of the pin is formed on the deposition layer on the disk. If no shadow appears on the deposition layer, this suggests that the deposition layer was formed isotropically. The probe can be applied to plasma-wall interaction studies in fusion devices and laboratory plasma devices such as linear divertor simulators to reveal the material migration mechanisms in such devices. The directional material probe method has been applied to plasma-wall interaction studies in the Large Helical Device (LHD), and a position-dependent variation in the directionality of deposition layer formation was found.
\end{abstract}

(c) 2013 The Japan Society of Plasma Science and Nuclear Fusion Research

Keywords: directional material probe, deposition layer, directionality, plasma-wall interaction, material migration

DOI: $10.1585 /$ pfr.8.1202110

In fusion devices, plasma-facing components are eroded by interactions with the plasma. The eroded materials migrate and are deposited elsewhere, forming a deposition layer. An understanding of the properties and formation mechanism of the deposition layer is very important for future fusion reactors. These deposition layers can be a source of dust as they are peeled off and can be a large sink for fueling particles, i.e., deuterium and tritium [1]. In particular, tritium retention in plasma-facing components is a serious problem in fusion reactors because tritium breeding rate will be limited to slightly greater than 1 [2]. In terms of safety, dust reduction is very important [3].

Some directionality has been observed in deposition layers in fusion devices. For example, in JT-60U, a carbon deposition layer was formed on the divertor tile in the inner divertor with directionality [4]. A deposition layer with directionality was also observed in LHD [5]. These directionalities showed the incident angle of the deposits, and the transport mechanism was deduced from the angle. Therefore, to understand the mechanisms of deposition layer formation, it is necessary to know the directionality of the layer, and the data should be taken at various positions in the vacuum vessel in fusion devices to understand the in-vessel material migration.

In the examples described above, the directionalities were analyzed by scanning electron microscopy (SEM) in JT-60U and transmission electron microscopy (TEM) in LHD, respectively. The observations take time not only for the observation itself but also for the fabrication of samples, such as focused ion beam fabrication [5]. Therefore, they are not necessarily suitable for many sample analyses focusing on directionality. On the other hand, visible shadows on the deposition layer are frequently observed

author'se-mail: masuzaki@LHD.nifs.ac.jp on plasma-facing components after plasma experiments. These shadows are cast by ledges onto the plasma-facing components. At times, the incident direction of the deposits can be determined by analyzing the shadows. However, it is difficult to remove plasma-facing components for detailed analysis with surface analysis devices.

The concept of the directional material probe (DMP) is depicted in Fig. 1. The DMP consists of a flat disk and shading pin. If deposits arrive at the DMP from a particular direction, a shadow of the pin is formed on the deposition layer on the disk. If deposits arrive isotropically, no shadow is formed. Thus, the directionality can be analyzed much more easily and quickly by visual observation and direct measurement than by SEM and/or TEM. Thus, many DMPs installed at various positions in the vacuum vessel of a fusion device can be analyzed to reveal material migration in the vacuum vessel. In addition to simple analysis with eyes and rulers, the DMP can be analyzed in detail using SEM, TEM, and other surface analysis methods, yielding a further understanding of the mechanism of the deposition layer formation.

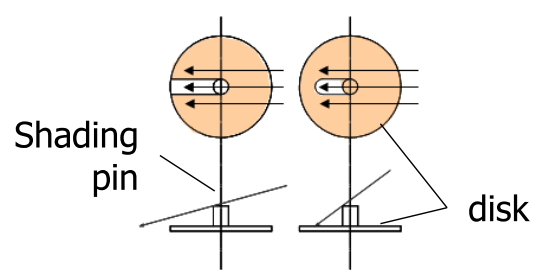

Fig. 1 Schematic of the directional material probe concept. The hatched parts represent the deposition layer. The arrows show the incident angles and directions of deposits. The two images show different incident angles of deposits. 


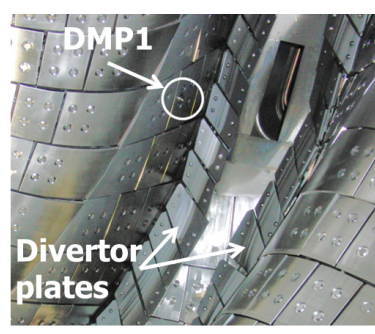

$q$
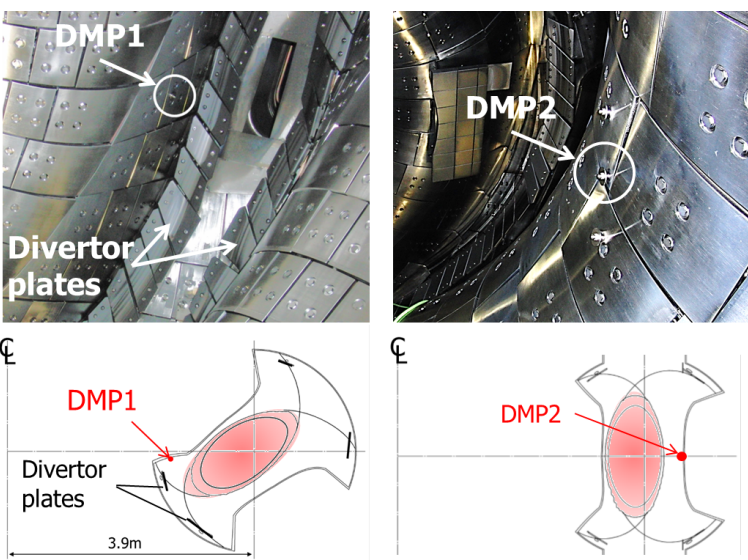

Fig. 2 Photos of the DMPs in the LHD vacuum vessel, and their positions in the poloidal cross-sections in the experimental campaign in 2010.

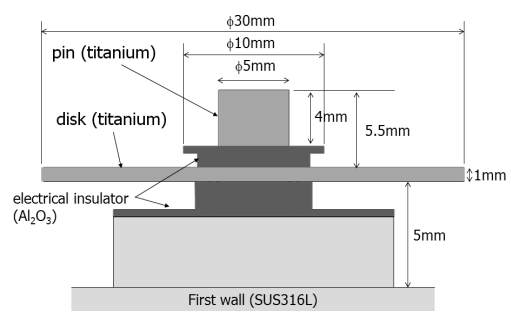

Fig. 3 Schematic of the DMP.

The DMPs were installed on the plasma-facing surfaces in the LHD vacuum vessel before the experimental campaign in 2010 as a trial. In the LHD vacuum vessel, the first wall panels are made of stainless steel (SUS316L) and the divertor plates are made of isotropic graphite. Two of the DMPs are shown in Fig. 2. One of them (DMP1) was installed on the first wall near the divertor plates on the torus-inboard side, and the other (DMP2) was installed on the first wall in a vertically elongated poloidal crosssection on the mid-plane on the torus-outboard side.

Figure 3 shows the schematic of the DMP. The diameter of the DMP's disk and shading pin were $30 \mathrm{~mm}$ and $5 \mathrm{~mm}$, respectively, and they were made of titanium. The disk was electrically insulated from the vacuum vessel to avoid erosion due to glow discharge for wall conditioning. One of the insulators was on the disk surface; its diameter was $10 \mathrm{~mm}$. The surface of the disk was about $6 \mathrm{~mm}$ above the first wall.

The DMPs were removed from the vacuum vessel after the experimental campaign. Figures 4 (a) and (b) show the photos of DMP1 and DMP2, respectively. Each photo shows the directions of the magnetic field lines on each surface. On the surface of DMP1, a clear shadow can be

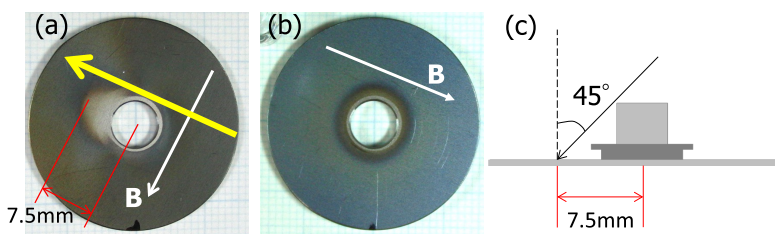

Fig. 4 Photos of the surfaces of (a) DMP1 and (b) DMP2 after the experimental campaign. The white arrows indicate the direction of magnetic field lines at each DMP's position. (c) Incident angle of deposits revealed from the shadow in the deposition on the surface of DMP1.

observed in the deposition. The direction shown by the yellow arrow in Fig. 4 (a) is almost perpendicular to the direction of the magnetic field lines. This suggests that the deposits were not carried by plasma flow along the field lines. The length of the shadow is $7.5 \mathrm{~mm}$. As depicted in Fig. 4 (c), the incident angle of the deposits on DMP1 can be estimated by the length of the shadow, and it is about $45^{\circ}$. This incident angle is similar to the angle estimated by the observation of the cross-section of the deposition layer on the material probe with TEM in Ref. [5]. The material probe in Ref. [5] was installed at a similar position to DMP1. In the material probe case, the dominant deposit was carbon, and its source was considered to be the divertor plates near the material probe. The deposition layer on the material probe was considered to be formed by direct deposition of sputtered carbon from the divertor plates, and this was qualitatively confirmed by a simulation using the ERO code [6]. For DMP1, the mechanism of deposition layer formation is considered to be the same as in the material probe case. On the other hand, no clear shadow can be observed on the surface of DMP2. This suggests that the deposits arrived at the surface isotropically.

The mapping of the directionality of deposition layer formation in the LHD vacuum vessel and more detailed analyses using surface analysis methods are in progress.

The author would like to thank the LHD experiment group and the technical staff of LHD for their support of this work. The author would also like to thank Drs. N. Yoshida, H. Watanabe, Y. Hatano and M. Tokitani for fruitful discussions. This work is supported in part by a Grantin-aid for Scientific Research (23656579) from MEXT, Japan.

[1] M. Shimada et al., J. Nucl. Mater. 438, S996 (2013).

[2] M. Nishikawa, Fusion Sci. Technol. 59, 350 (2011).

[3] J. Roth, J. Nucl. Mater. 390-391, 1 (2009).

[4] Y. Gotoh et al., J. Nucl. Mater. 313-316, 370 (2003).

[5] M. Tokitani et al., J. Nucl. Mater. 417, 668 (2011).

[6] G. Kawamura et al., Contrib. Plasma Phys. 50, 451 (2010). 